\title{
Wavelength optimization for rapid chromophore mapping using spatial frequency domain imaging
}

\author{
Amaan Mazhar \\ University of California \\ Department of Biomedical Engineering \\ and \\ Beckman Laser Institute, and Medical Clinic \\ Irvine, California 92612
}

\section{Steven Dell}

University of California

Beckman Laser Institute and Medical Clinic Irvine, California 92612

\section{David J. Cuccia}

Modulated Imaging Incorporated

Technology Incubator Office

Irvine, California 92612

\author{
Sylvain Gioux \\ Boston Univeristy \\ Department of Biomedical Engineering \\ Boston, Massachusetts 02215 \\ and \\ Beth Israel Deaconess Medical Center \\ Division of Hematology/Oncology \\ Department of Medicine \\ Boston, Massachusetts 02215
}

\author{
Anthony J. Durkin \\ University of California \\ Beckman Laser Institute and Medical Clinic \\ Irvine, California 92612
}

\section{John V. Frangioni}

Beth Israel Deaconess Medical Center

Division of Hematology/Oncology,

Department of Medicine

and

Department of Radiology,

Boston, Massachusetts 02215

\section{Bruce J. Tromberg}

University of California

Department of Biomedical Engineering and

Beckman Laser Institute, and Medical Clinic Irvine, California 92612

\begin{abstract}
Spatial frequency-domain imaging (SFDI) utilizes multiplefrequency structured illumination and model-based computation to generate two-dimensional maps of tissue absorption and scattering properties. SFDI absorption data are measured at multiple wavelengths and used to fit for the tissue concentration of intrinsic chromophores in each pixel. This is done with a priori knowledge of the basis spectra of common tissue chromophores, such as oxyhemoglobin $\left(\mathrm{ctO}_{2} \mathrm{Hb}\right)$, deoxyhemoglobin $(\mathrm{ctHHb})$, water $\left(\mathrm{ctH}_{2} \mathrm{O}\right)$, and bulk lipid. The quality of in vivo SFDI fits for the hemoglobin parameters $\mathrm{ctO}_{2} \mathrm{Hb}$ and $\mathrm{ctH} H \mathrm{Hb}$ is dependent on wavelength selection, fitting parameters, and acquisition rate. The latter is critical because SFDI acquisition time is up to six times longer than planar two-wavelength multispectral imaging due to projection of multiple-frequency spatial patterns. Thus, motion artifact during in vivo measurements compromises the quality of the reconstruction. Optimal wavelength selection is examined through matrix decomposition of basis spectra, simulation of data, and dynamic in vivo measurements of a human forearm during cuff occlusion. Fitting parameters that minimize cross-talk from additional tissue chromophores, such as water and lipid, are determined. On the basis of this work, a wavelength pair of $670 \mathrm{~nm} / 850 \mathrm{~nm}$ is determined to be the optimal two-wavelength combination for in vivo hemodynamic tissue measurements provided that assumptions for water and lipid fractions are made in the fitting process. In our SFDI case study, wavelength optimization reduces acquisition time over 30 -fold to $1.5 \mathrm{~s}$ compared to 50 s for a full 34-wavelength acquisition. The wavelength optimization enables dynamic imaging of arterial occlusions with improved spatial resolution due to reduction of motion artifacts. $\odot 2010$ Society of Photo-Optical Instrumentation Engineers. [DOI: 10.1117/1.3523373]
\end{abstract}

Keywords: multispectral imaging; diffuse optical spectroscopy; hemodynamics.

Paper 10027SSR received Feb. 3, 2010; revised manuscript received Oct. 7, 2010; accepted for publication Oct. 14, 2010; published online Dec. 23, 2010.

\section{Introduction}

The propagation of multiply scattered near-infrared (NIR) light is particularly sensitive to absorption loss in microvascular structures. As a result, NIR spectroscopy (NIRS) and imaging are

Address all correspondence to: Bruce J. Tromberg, University of California, Beckman Laser Institute and Medical Clinic, 1002 Health Sciences Road, Irvine, California 92612; Tel: 949-824-8705; E-mail: bjtrombe@uci.edu widely used to measure hemodynamic changes in tissues such as brain, breast, muscle, and skin. ${ }^{1-5}$ In the NIR spectral region, $(\sim 650-1000 \mathrm{~nm})$, the primary sources of endogenous absorption (i.e., molecular) contrast are oxy-hemoglobin $\left(\mathrm{ctO}_{2} \mathrm{Hb}\right)$, deoxy-hemoglobin (ctHHb), water, and lipids. In NIRS and related methods, such as diffuse optical spectroscopy, the wavelength dependence of light remitted from the tissue, combined

1083-3668/2010/15(6)/061716/9/\$25.00 (C) 2010 SPIE 
with knowledge of pure component spectra, is used to calculate tissue chromophore content. Measurement accuracy and precision are influenced by the source spectral bandwidth and the tissue structural and scattering properties. As a result, there is a trade-off between quantitative accuracy and measurement acquisition time that impacts our ability to rapidly measure and image dynamic physiological processes.

NIRS measurements are typically based on time-independent, continuous-wave (CW) techniques that do not separate light absorption from scattering. Assumptions are made regarding the reduced scattering parameter $\left(\mu^{\prime}\right)$ to estimate the optical path length. A modified Beer-Lambert law is used to fit for chromophores in these cases. ${ }^{6}$ Because the BeerLambert law is a linear algebraic fit, the number of spectral points required is equivalent to the number of chromophores to be measured. However, assumptions made about optical path length can result in cross talk and errors in hemodynamic fits. Metrics for understanding this cross talk between $\mathrm{ctO}_{2} \mathrm{Hb}$ and ctHHb have been discussed in great detail, ${ }^{7-9}$ and studies have shown that two optimized wavelengths $(690 \mathrm{~nm} / 830$ $\mathrm{nm})$ minimize errors when monitoring relative hemodynamic changes. $^{10-12}$

Diffuse optical tomography (DOT) is an imaging technique that reconstructs chromophore concentration in three dimensions. ${ }^{13,14}$ DOT requires additional spectral and/or temporal content to overcome the nonlinearity associated with separating absorption from scattering. This technique is typically used in breast measurements, where hemoglobin, lipid, and water are chromophores of interest. Optimized spectral bands show that five wavelengths are needed to extract four chromophore concentrations and to account for scattering amplitude. ${ }^{13}$ In this domain, wavelength band optimization has been shown to reduce computational requirements while maintaining and sometimes improving accuracy in reconstruction values. ${ }^{15}$

Methods that separately quantify tissue optical absorption and reduced scattering parameters ( $\mu_{\mathrm{a}}$ and $\mu_{\mathrm{s}}^{\prime}$, respectively) can eliminate the need to approximate optical path length and to fit for scattering. In general, these methods modulate the light source and compare measurements to a mathematical model of light propagation in tissue. Time-domain systems characterize the temporal point-spread function of an ultrafast light pulse, ${ }^{16}$ while frequency-domain systems measure the amplitude and phase of diffusely propagating $\mathrm{MHz}-\mathrm{GHz}$ photon density waves. ${ }^{17}$ In order to generate images, these methods typically require source scanning and/or multiple source-detector views combined with relatively complex time- and frequencydomain components.

Spatial frequency-domain imaging (SFDI) is an alternative to time-resolved methods for separating absorption from scattering in tissue. SFDI utilizes a spatial light modulator to project structured light patterns onto tissue. By imaging the frequencydependent reflectance at multiple wavelengths and comparing to light propagation models, absorption and scattering can be calculated in every pixel of an SFDI image. SFDI employs a CCD camera in a noncontact wide-field imaging geometry and is compatible with optical property mapping and subsurface tomography. ${ }^{18,19}$

Although SFDI separates absorption from scattering, multispectral measurements are required in order to accurately map relevant tissue chromophores, such as $\mathrm{ctO}_{2} \mathrm{Hb}$ and ctHHb.
However, no objective criteria have been established using this method to determine the trade-off between reducing spectral content and maintaining accuracy in chromophore fitting. Consequently, spectral data are often over acquired at the cost of temporal resolution in order to ensure accurate chromophore fits. An acquisition scheme that minimizes spectral content could improve SFDI temporal resolution, enable observation of physiological events, and reduce errors due to motion artifacts in vivo.

In this study, we describe an approach for understanding the multidimensional parameter space associated with obtaining accurate hemodynamic maps during SFDI. First, we define a general metric for determining optimal spectral bands, based on the work presented by Corlu et al. ${ }^{14}$ Second, the impact of confounding intrinsic chromophores in our fitting methods is investigated. Third, a reduced spectral parameter space is applied to in vivo measurements. Finally, the impact of light-source spectral bandwidth properties on fits is explored. Importantly, the analysis we present can be extended to any imaging or spectroscopic platform that is capable of extracting absorption coefficient values from turbid media.

\section{Material and Methods}

\subsection{Instrumentation}

SFDI consists of three basic components: a light source, spatial light modulator (SLM), and a CCD camera. A 250-Watt broadband quartz tungsten halogen lamp (Newport Corporation, Irvine, California) is directed onto a DLP Developers Kit (Texas Instruments, Dallas, Texas) consisting of a 1024 $\times 768$ pixel digital micromirror device (DMD). The DMD creates patterns that are projected onto the imaging surface. The patterns on the chip are controlled by customized C\# software, and the projection is imaged by a set of lenses onto the surface of the sample at a working distance of $500 \mathrm{~mm}$. Cross polarizers are implemented at the source and detector to eliminate specular reflection and capture diffusely reflected light. A Nuance Multipsectral Imaging System (CRI Inc., Woburn, Massachusetts), which contains a liquid-crystal tunable filter, is used for multispectral data acquisition between 650 and $980 \mathrm{~nm}$ with $10-\mathrm{nm}$ resolution. Chromophore fits for oxy-hemoglobin, deoxy-hemoglobin, lipid, and water are generally obtained by taking spectral band data at 34 wavelengths from 650 to 980 $\mathrm{nm}$. Because of the number of spatial frequencies $(0$ and 0.15 $\left.\mathrm{mm}^{-1}\right)$ and phase offset measurement $(0,120,240 \mathrm{deg})$, a total of up to 204 images ( 2 spatial frequencies $\times 3$ phases $\times 34$ wavelengths) are captured for a single multispectral frame lasting $\sim 50$ s. The image at each wavelength is autoexposed to use the full dynamic range of the camera. Because of the spectral characteristics of the light source and reduced quantum efficiency of the $\mathrm{CCD}$, longer wavelengths have greater exposure times $(\sim 1200$ $\mathrm{ms}$ at $980 \mathrm{~nm})$ than shorter wavelengths $(100 \mathrm{~ms}$ at $650 \mathrm{~nm})$. Thus, it takes $\sim 9$ s to acquire 34 evenly spaced images across the full wavelength spectrum of the system $(650-980 \mathrm{~nm})$. In comparison, a two-wavelength imaging scheme can reduce acquisition time for hemodynamic measurements so that only 12 images are needed ( 2 spatial frequencies $\times 3$ phases $\times 2$ wavelengths). These wavelengths can be at higher throughput wavelengths, and the temporal resolution is $1.5 \mathrm{~s} /$ frame. All image 
processing was carried out using Matlab software (Mathworks, Natick, Massachusetts).

\subsection{Optical Property Mapping with SFDI}

The theory behind tissue optical property characterization and imaging in the spatial frequency domain has been described. ${ }^{18-20}$ SFDI uses spatially modulated structured illumination patterns projected onto tissue at various frequencies in the following form:

$$
S=\frac{S_{o}}{2}\left[1+M_{0} \cos \left(2 \pi f_{x}+\alpha\right)\right],
$$

where $S_{0}, M_{0}, f_{\mathrm{x}}$, and $\alpha$ are the illumination source intensity, modulation depth, spatial frequency, and spatial phase, respectively. The remitted light, or diffuse reflectance, from the sample is then captured by a CCD camera. This reflected light, $I$, is composed of ac and dc components:

$$
I=I_{\mathrm{ac}}+I_{\mathrm{dc}} .
$$

The measured ac component of the reflected intensity, $I_{\mathrm{ac}}$, can be characterized as

$$
I_{\mathrm{ac}}=M_{\mathrm{ac}}\left(x, f_{x}\right) * \cos \left(2 \pi f_{x}+\alpha\right),
$$

where $M_{\mathrm{ac}}(x, f x)$ represents the modulation of diffusively reflected photon density waves (PDW). PDW modulation in turbid media depends on the optical properties of the tissue and can be modeled with diffusion-based or Monte Carlo-based light-transport methods. In order to extract $M_{\mathrm{ac}}(x, f x)$, a signal demodulation method is used. The sample is illuminated with a sinusoidal pattern at a specific spatial frequency with three phase offsets $\alpha=0,2 \pi / 3$, and $4 \pi / 3$ radians. $M_{\mathrm{ac}}(x, f x)$ is then calculated using the demodulation expression,

$$
\begin{aligned}
M_{\mathrm{ac}}\left(x, f_{x}\right)= & \frac{\sqrt{2}}{3}\left[\left(I_{1}-I_{2}\right)^{2}+\left(I_{2}-I_{3}\right)^{2}\right. \\
& \left.+\left(I_{3}-I_{2}\right)^{2}\right]^{1 / 2} .
\end{aligned}
$$

In order to calculate tissue optical properties, $M_{\mathrm{ac}}\left(x, f_{\mathrm{x}}\right)$ can be measured at two spatial frequencies, $f_{x}$, and then fit using a lookup table (LUT) created with a known forward Monte Carlo model. ${ }^{19}$ The present study utilizes a single high spatial frequency $\left(0.15 \mathrm{~mm}^{-1}\right)$ measurement combined with a low-frequency measurement $\left(0 \mathrm{~mm}^{-1}\right)$ to calculate $\mu_{\mathrm{a}}$ and $\mu_{\mathrm{s}}^{\prime}$. First, multiple-frequency patterns are projected over the sample [Fig. 1(a)]. The patterns are projected at multiple phases over the sample and then demodulated using Eq. (4) (not shown). Next, the diffuse reflectance is calculated at each spatial frequency by calibrating to a silicone calibration phantom of known optical properties [Fig. 1(b)]. This calibration corrects for any system response due to spatial variation of the illumination light and lens aberrations. Finally, a LUT-based fit is applied to all pixels in the image in order to generate optical property maps at discrete wavelengths [Fig. 1(c)].

In order to measure intrinsic biological chromophores, such as oxy-hemoglobin, deoxy-hemoglobin, water, and lipid, the scattering corrected optical absorption maps are measured at discrete wavelengths using SFDI. In general, the optical absorption at a single wavelength, $\lambda_{i}$, can be characterized by (a)
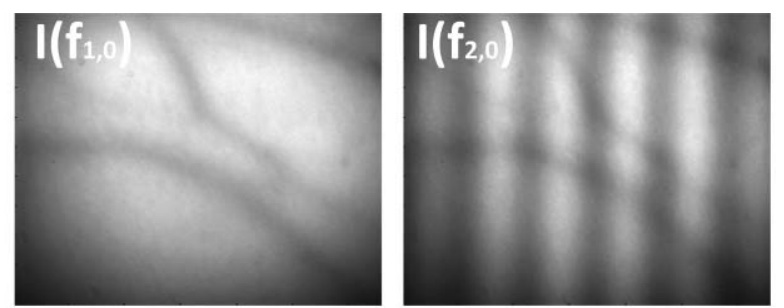

(b)
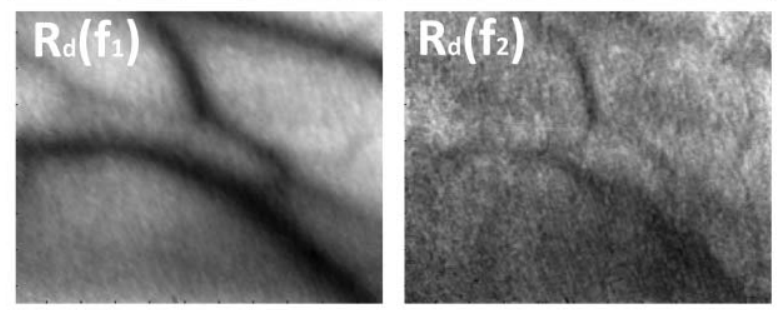

(c)
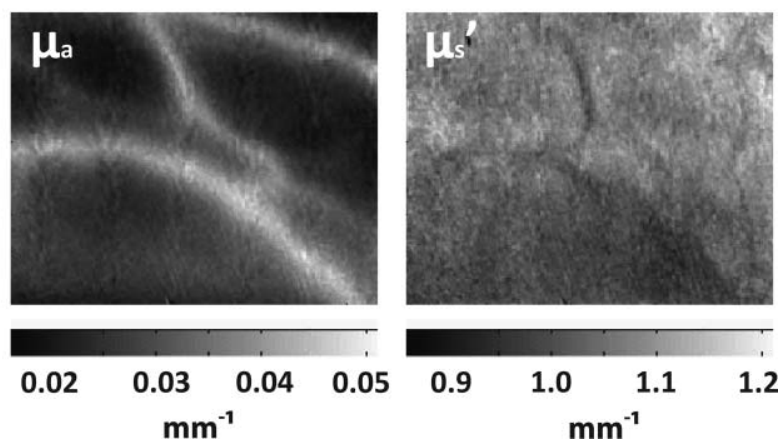

Fig. 1 SFDI data flow. (a) Multiple frequencies $\left(f_{x}\right)$ are projected onto the sample, and remitted intensity $I$ is captured. (b) Each illumination frequency is imaged at three phases, then demodulated and calibrated to yield $R_{\mathrm{d}}$. (c) The $R_{\mathrm{d}}$ at each pixel is fit for $\mu_{\mathrm{a}}$ and $\mu_{\mathrm{s}}^{\prime}$ using a Monte Carlo light-transport model and lookup table to yield optical property maps.

$$
\mu_{a}\left(\lambda_{i}\right)=\sum_{n=1}^{N} \varepsilon_{n}\left(\lambda_{i}\right) c_{n}
$$

where $\mu_{\mathrm{a}}, \varepsilon_{n}\left(\lambda_{i}\right), \mathrm{c}_{n}$, and $N$ represent the optical absorption coefficient, the chromophore extinction coefficient at a given wavelength, the chromophore concentration, and the number of chromophores, respectively. With prior knowledge of the extinction coefficient matrix, $\varepsilon_{n}\left(\lambda_{i}\right)$, and measurements of the absorption coefficient at multiple wavelengths, the concentration $\mathrm{c}_{n}$ of each chromophore can be extracted using the following matrix operation:

$$
\left[\begin{array}{c}
\mu_{\mathrm{a}}\left(\lambda_{1}\right) \\
\vdots \\
\mu_{\mathrm{a}}\left(\lambda_{j}\right)
\end{array}\right]=\left[\begin{array}{ccc}
\varepsilon_{1}\left(\lambda_{1}\right) & \cdots & \varepsilon_{i}\left(\lambda_{1}\right) \\
\vdots & \ddots & \vdots \\
\varepsilon_{1}\left(\lambda_{j}\right) & \cdots & \varepsilon_{i}\left(\lambda_{j}\right)
\end{array}\right] *\left[\begin{array}{c}
c_{1} \\
\vdots \\
c_{i}
\end{array}\right],
$$

where $i$ and $j$ represent the number of chromophores and number of measured wavelengths, respectively. It is important to note that the problem requires that $j \geq i$ in order to adequately separate the chromophore concentration values. Typical basis spectra for tissue chromophores have been measured and characterized by multiple researchers and are used here [Fig. 2]. ${ }^{21,22}$ 


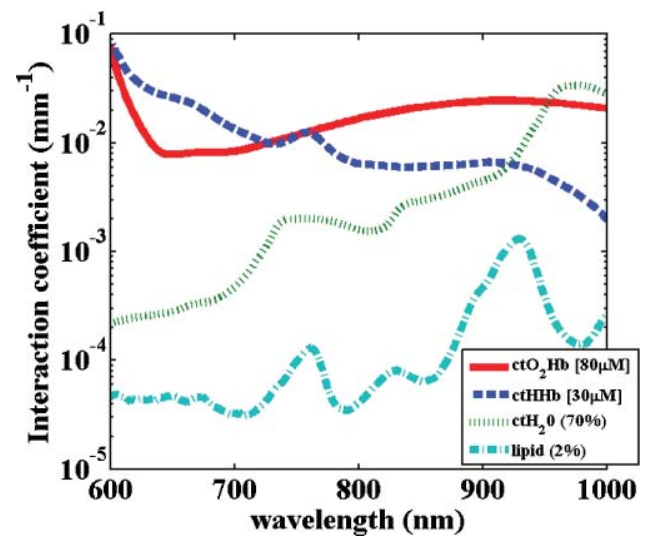

Fig. 2 Common tissue chromophores and their basis spectra for typical concentrations and volume fractions.

\subsection{Condition Number Analysis}

Corlu et al. have demonstrated a two-step method to determine optimal wavelengths in $\mathrm{CW}$ diffuse optical imaging. First, a singular value decomposition of the extinction coefficient matrix gives a measure of the contribution of each chromophore to the overall absorption. ${ }^{14}$ The condition number, $\kappa(\varepsilon)$, of the extinction coefficient matrix [first term on the right-hand side of Eq. (6)] is defined as the ratio of the maximum and minimum singular values (SVD) of the matrix;

$$
\kappa=\frac{\mathrm{SVD}_{\max }}{\mathrm{SVD}_{\min }} \text {. }
$$

The assertion is that the lower the $\kappa$ is, the more accurate the fit of the chromophores due to equivalent sensitivity to the chromophores in question. ${ }^{14}$ Second, a residual value is calculated to evaluate the nonuniqueness of the solutions in $\mathrm{CW}$ imaging due to variability in scattering amplitude. ${ }^{23}$ In this case, SFDI is able to calculate the scattering amplitude and nonuniqueness is no longer an issue in the measurement. Thus, the residual is not calculated or optimized in this study.

\subsection{In Vivo Measurements}

In vivo measurements were taken of a human wrist under an approved UC Irvine institutional review board protocol. All SFDI measurements were performed at two spatial frequencies ( 0 and $0.15 \mathrm{~mm}^{-1}$ ) and a LUT method was used to calculate the optical properties. Initially, measurements were taken at 34 wavelengths evenly spaced between 650 and $980 \mathrm{~nm}$. The recovered absorption maps at each wavelength were used to extract the chromophore concentrations. The data were then decomposed from 34 wavelengths to a few specific wavelength combinations $(670 \mathrm{~nm} / 850 \mathrm{~nm}, 730 \mathrm{~nm} / 850 \mathrm{~nm}, 710 \mathrm{~nm} / 980 \mathrm{~nm}$, $670 \mathrm{~nm} / 850 \mathrm{~nm} / 980 \mathrm{~nm}$, and $670 \mathrm{~nm} / 850 \mathrm{~nm} / 920 \mathrm{~nm} /$ $980 \mathrm{~nm})$. The mean $\mathrm{cto}_{2} \mathrm{Hb}$ and $\mathrm{ctHHb}$ fits for the image were compared to a 34-wavelength fit. In order to demonstrate the improvement in temporal resolution, the optimal wavelength pairs were used to perform a hemodynamic measurement of an arterial occlusion. A patient wrist was measured for $10 \mathrm{~min}$. After $1 \mathrm{~min}$, an arm cuff with $240-\mathrm{mm} \mathrm{Hg}$ pressure was applied to the bicep in order to create an arterial occlusion. The occlusion was held in place for $4 \mathrm{~min}$ before the pressure was released. Chromophore maps were plotted at all time points, and a region of interest (ROI) was selected to show mean changes in tissue hemodynamic content over time.

\section{Results}

\subsection{Simulation of In Vivo Data}

Matlab code was generated to plot the condition number of oxy- and deoxy-hemoglobin for all wavelength pairs at $1 \mathrm{~nm}$ intervals ranging from 650 to $1000 \mathrm{~nm}$. Basis spectra for ctO $\mathrm{O}_{2} \mathrm{Hb}$ and $\mathrm{ctHHb}$ were used from previously published literature. ${ }^{22}$ The contour plot shows a minimum condition number of 2.21 at wavelength pairs 712 and $980 \mathrm{~nm}$ when water and lipid are not included in simulation and fit [Fig. 3(a)]. The graph for condition number is symmetric on the diagonal axis because it shows a metric for the combined fitting stability of $\mathrm{ctO}_{2} \mathrm{Hb}$ and ctHHb. It should also be noted that the condition numbers surrounding these wavelength regions are all within $25 \%$ of this value. These condition numbers were then compared to simulated absorption spectrum generated from 650 to 1000 $\mathrm{nm}$ using typically reconstructed $\mathrm{ctO}_{2} \mathrm{Hb}$ and ctHHb concentrations (80 and $30 \mu \mathrm{M}$, respectively). Gaussian noise (FWHM $10 \%$ ) was added randomly to the simulated absorption data in order to replicate a real measurement, and the procedure was repeated 100 times. $\mathrm{ctO}_{2} \mathrm{Hb}$ and ctHHb concentrations were reconstructed using wavelength pairs in $1 \mathrm{~nm}$ intervals. Unlike the condition number analysis, these simulations allow for percent error calculation for each chromophore. The plots are no longer symmetric, as in Fig. 3(a) as $\mathrm{ctO}_{2} \mathrm{Hb}$ (lower right) and ctHHb (upper left) are evaluated seperately. The absolute percent error [Fig. 3(b)] from simulated data maps results in comparable regions of minimized error to the condition number, with an absolute minimum at 710 and $972 \mathrm{~nm}$. This variation from the (a) Kappa

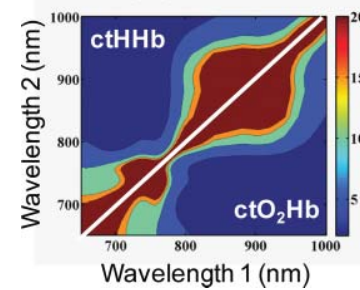

(b) \% error

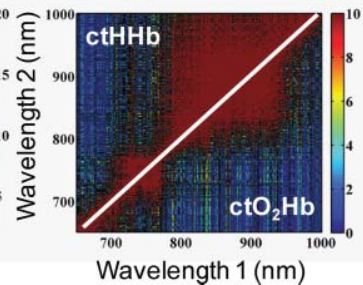

(c) COV

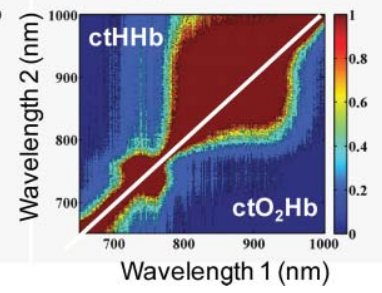

Fig. 3 Condition number and simulation of hemodynamics: (a) Condition number $(\kappa)$ plot for $\mathrm{ctO}_{2} \mathrm{Hb}$ and ctHHb. Lower condition number indicates acceptable wavelength combination space. (b) Spectral simulations show average percent error in $\mathrm{ctO}_{2} \mathrm{Hb}$ and ctHHb fits for wavelength pairs when water and lipid are not considered in the calculation. (c) COV of the same simulation shows stability of a single measurement for each wavelength pair. 
(a) Kappa

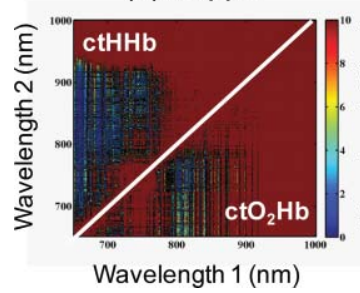

(b) \% error

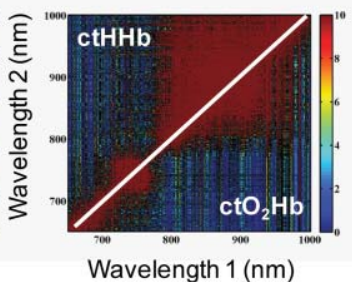

(c) $\mathrm{COV}$

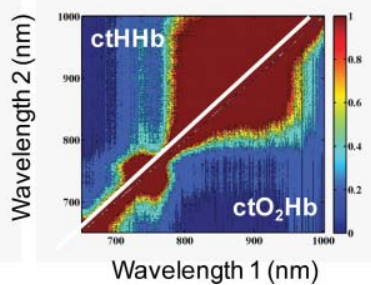

Fig. 4 Simulation showing impact of other tissue chromophores (i.e., water and lipid) on hemodynamic fitting. (a) Average percent error for in $\mathrm{ctO}_{2} \mathrm{Hb}$ and ctH Hb for all wavelength pairs when no assumptions for water and lipid are made limits the acceptable wavelength combination space, with error values of $<10 \%$. (b) Making assumptions for water (50\%) and lipid ( $2 \%$ ) enlarges the acceptable space as shown by the mean percent error. (c) COV for this fit shows the wavelength space with the most stable measurements.

condition number can be attributed to simulation noise. The coefficient of variation (COV) map [Fig. 3(c)] is a measure of measurement stability and indicates that the same wavelength space produces the most stable results (lowest covariance) in the case of a single measurement. The condition number plot and the simulations are consistent with the consensus opinion in the field that a two wavelength system works best when there is one wavelength above and one wavelength below $800 \mathrm{~nm}$.

In reality, tissue consists of many contributing chromophores in the NIR: $\mathrm{ctO}_{2} \mathrm{Hb}$, ctHHb, water, and lipid. In order to study the impact of other chromophores, 50\% volume fraction of water and $2 \%$ volume fraction of lipid were added to typical oxy- and deoxy-hemoglobin concentrations (80 and $30 \mu \mathrm{M}$, respectively) to generate absorption spectrum. Water content in skin is shown to vary between 25 and $75 \%$ for normal skin; thus $50 \%$ was chosen as a central fitting parameter to demonstrate water-independent fitting of hemodynamics. ${ }^{24,25}$ Lipid values also vary from 0 to $50 \%$ based on tissue type, location, and individual. ${ }^{3}$ In vivo measurements in this study are taken in the wrist, where subcutaneous fat contributions are low. Thus, a representative value of $2 \%$ is assumed for simulations. First, fitting was performed for $\mathrm{ctO}_{2} \mathrm{Hb}$ and ctHHb in order to determine the effect of confounding chromophores [Fig. 4(a)]. Simulations show that water and lipid content in tissue can lead to large errors
(> 10\%) for hemodynamic fits. The optimal region in this simulation includes a first wavelength between 670 and $780 \mathrm{~nm}$ and second wavelength region between 800 and $850 \mathrm{~nm}$. Second, a two-wavelength fit was performed using assumed fixed values for water (50\%) and lipid (2\%). Simulations reveal that the error [Fig. 4(b)] can be centered so that the magnitude is much smaller $(<5 \%)$. This creates a much larger region of wavelength pairs that produce less than a $5 \%$ error on average. A plot of the $\mathrm{COV}$ for these simulations reveals the best wavelength regions for stable chromophore fits. The best regions occur when one wavelength is between 620 and $700 \mathrm{~nm}$ and the second is between 800 and $900 \mathrm{~nm}$ [Fig. 4(c)]. The local minimum for the $\mathrm{COV}$ occurs at the wavelength pair 670 and $850 \mathrm{~nm}$. The reason why $730 \mathrm{~nm}$ has a larger COV is that water contributes a little more to the overall signal and can further confound the spectra. There is also a distinct ctHHb peak closer to $730 \mathrm{~nm}$ in the basis spectrum. Thus, any deviations in the measurement can result in a larger error in the ctHHb fit at 730 versus $670 \mathrm{~nm}$.

Using the two wavelength pairs that showed the lowest percent error in the second case of simulations, water and lipid distribution was varied $0-100 \%$ in the forward spectrum and the impact using a 50\% and 2\% fixed parameter for fitting for hemodynamics was studied. Analysis of the water variation shows that the two sets of optimal wavelength pairs $(670 \mathrm{~nm} / 850 \mathrm{~nm}$ and (a)

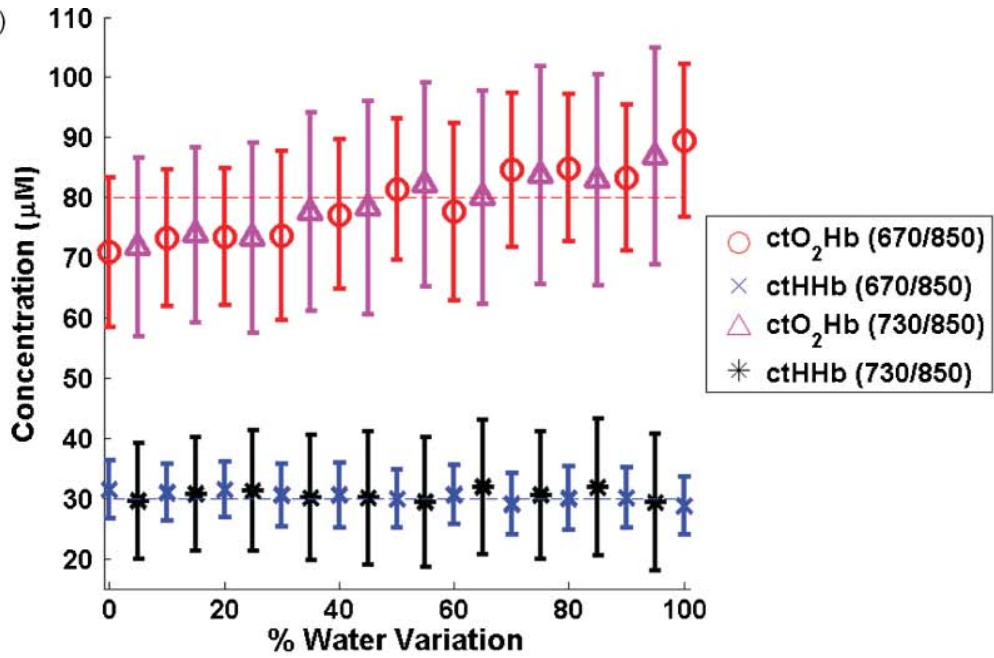

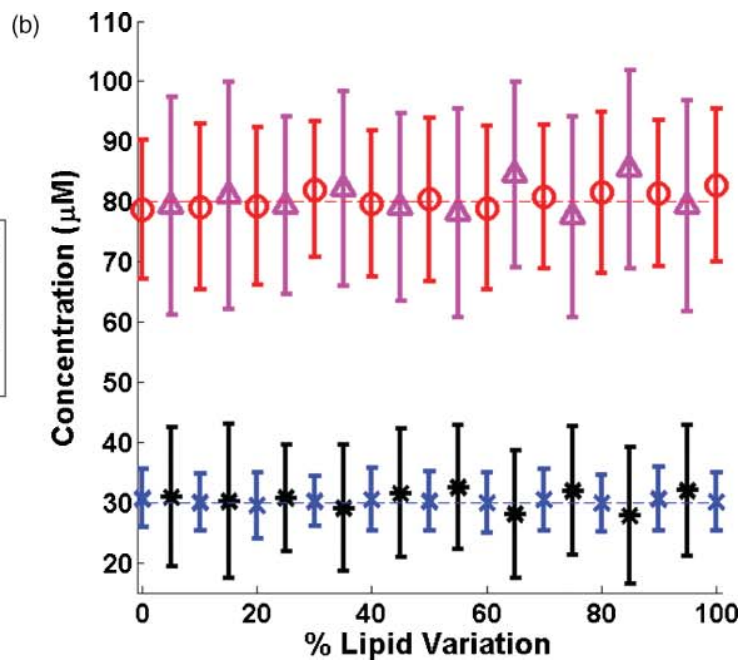

Fig. 5 Impact of water and lipid variation on hemodynamic fitting for two ideal wavelength pairs. System variation in (a) water fraction and (b) lipid fraction shows a $<10 \%$ error in mean hemodynamic fit using two common wavelength pairs (670/850 and 730/850). Both wavelength combinations perform the same in terms of mean, but the $670 / 850$ combination has lower standard deviations, indicating it is much more stable for single point measurements. 
$730 \mathrm{~nm} / 850 \mathrm{~nm}$ ) behave similarly in terms of fits when a fixed assumption (50\%) for water and lipid is used [Fig. 5(a)]. The mean percent error at the extremes is under $10 \%$ for $\mathrm{ctO}_{2} \mathrm{Hb}$ and under $5 \%$ for ctHHb when $10 \%$ Gaussian noise is added to the simulated signal. Depending on tissue type, water content in skin is shown to vary between 25 and $75 \%,{ }^{24,25}$ which reduces the expected error to $5 \%$ for $\mathrm{ctO}_{2} \mathrm{Hb}$ and $2.5 \%$ for $\mathrm{ctHHb}$. Variation of lipid content has an even smaller impact on fits for both these wavelengths combinations with the expected error always falling below 5\% [Fig. 5(b)]. The standard deviation of both simulations and wavelength combinations show a much larger variation for the $730 / 850 \mathrm{~nm}$ wavelength pair [Figs. 5(a), and 5(b)]. The COV for the $670 / 850$ pair is $\sim 0.15$ for all values of water. However, the COV is on average 0.22 and 0.35 for $\mathrm{ctO}_{2} \mathrm{Hb}$ and ctHHb, respectively, when using the $730 \mathrm{~nm} /$ $850 \mathrm{~nm}$ pair. This indicates that a single measurement will be much more stable when using the $670 \mathrm{~nm} / 850 \mathrm{~nm}$ combination.

\subsection{In Vivo Studies}

In vivo data for a human wrist shows the impact of reducing spectral acquisition data. A simple reflectance plot at approximately the isobestic point $(805 \mathrm{~nm})$ for hemoglobin shows the vein architecture in the wrist [Fig. 6(a)]. Hemodynamic images are generated using a full 34-wavelength acquisition and fitting procedure [Figs. 6(b), and 6(c)]. The maps generated when optimized spectral wavelengths are used result in images that have a $<10 \%$ variation in hemodynamic fits for all pixels in the image. Analysis of tissue ROI with decomposed spectral data show that the $670 \mathrm{~nm} / 850 \mathrm{~nm}$ pair with a fixed value of $50 \%$ for water performs the best hemodynamic fit compared to the full 34-wavelength fit [Fig. 6(d)]. Flat ROIs were chosen to limit the effect of surface curvature. A $730 \mathrm{~nm} / 850 \mathrm{~nm}$ combination results in a slight overestimation in ctHHb as compared to the full fit. A $670 \mathrm{~nm} / 850 \mathrm{~nm}$ hemodynamic fit with no water fit results in an overestimation in the mean value of $\mathrm{ctO}_{2} \mathrm{Hb}(10 \%)$. This is even more exaggerated $(80 \%)$ when considering the $710 \mathrm{~nm} / 980 \mathrm{~nm}$ combination that is predicted as optimal by the initial condition number plot. For more accurate fits, water fraction content should be assumed or calculated when calculating hemodynamic parameters in vivo.

The main benefit of reduced spectral content is improved temporal resolution. For this SFDI system, a typical 34-wavelength SFDI acquisition takes $50 \mathrm{~s}$ to acquire as opposed to $1.5 \mathrm{~s}$ for the two-wavelength fit. The imaging time has a nonlinear relationship due to nonuniform power distribution of the source spectrally. In addition, increased imaging time can lead to loss in spatial resolution due to patient motion. In order to demonstrate a time-sensitive measurement, an arterial occlusion was performed on a human patient to measure the tissue hemodynamics. $\mathrm{ctO}_{2} \mathrm{Hb}$ and $\mathrm{ctHHb}$ values were calculated using the $670 \mathrm{~nm} / 850 \mathrm{~nm}$ wavelength pair. Water and lipid fractions
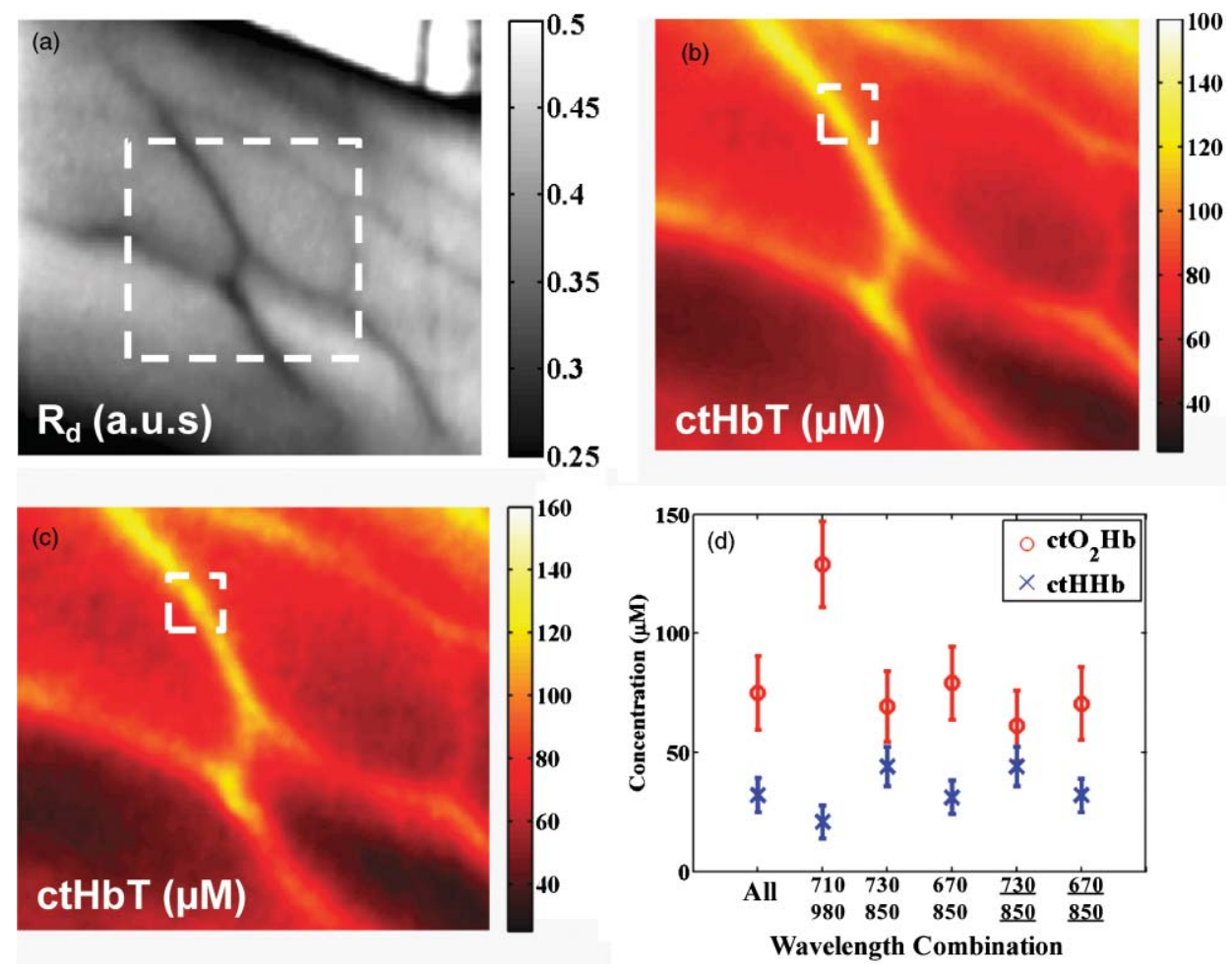

Fig. 6 Reduced spectral content maintains integrity of hemodynamic maps. (a) A single snapshot diffuse reflectance map at $800 \mathrm{~nm}$ shows high contrast for veins. (b) SFDI chromophore map produced by a 34-wavelength fit produces comparable maps to (c) two-wavelength fit. The mean and standard deviation of a hemodynamic is calculated over a small ROI covering a vein with multiple wavelength combinations. Wavelength combinations include (1) 34 evenly spaced wavelengths between 650 and $980 \mathrm{~nm}$ (all), (2) $710 \mathrm{~nm} / 980 \mathrm{~nm}$, (3) $730 \mathrm{~nm} / 850 \mathrm{~nm}$, (4) $670 \mathrm{~nm} / 850 \mathrm{~nm}$, (5) $670 \mathrm{~nm} / 850 \mathrm{~nm}$ and (6) $730 \mathrm{~nm} / 850 \mathrm{~nm}$. Combination 1 is fit for four chromophores. Combinations 2-4 are fit for hemodynamic values, with no assumption for water fraction. Underlined combinations 5 and 6 assume a fixed water fraction of $50 \%$ to improve fits for hemodynamic parameters. A $670 \mathrm{~nm} / 850 \mathrm{~nm}$ pair with assumed water and lipid fractions performs the best. 

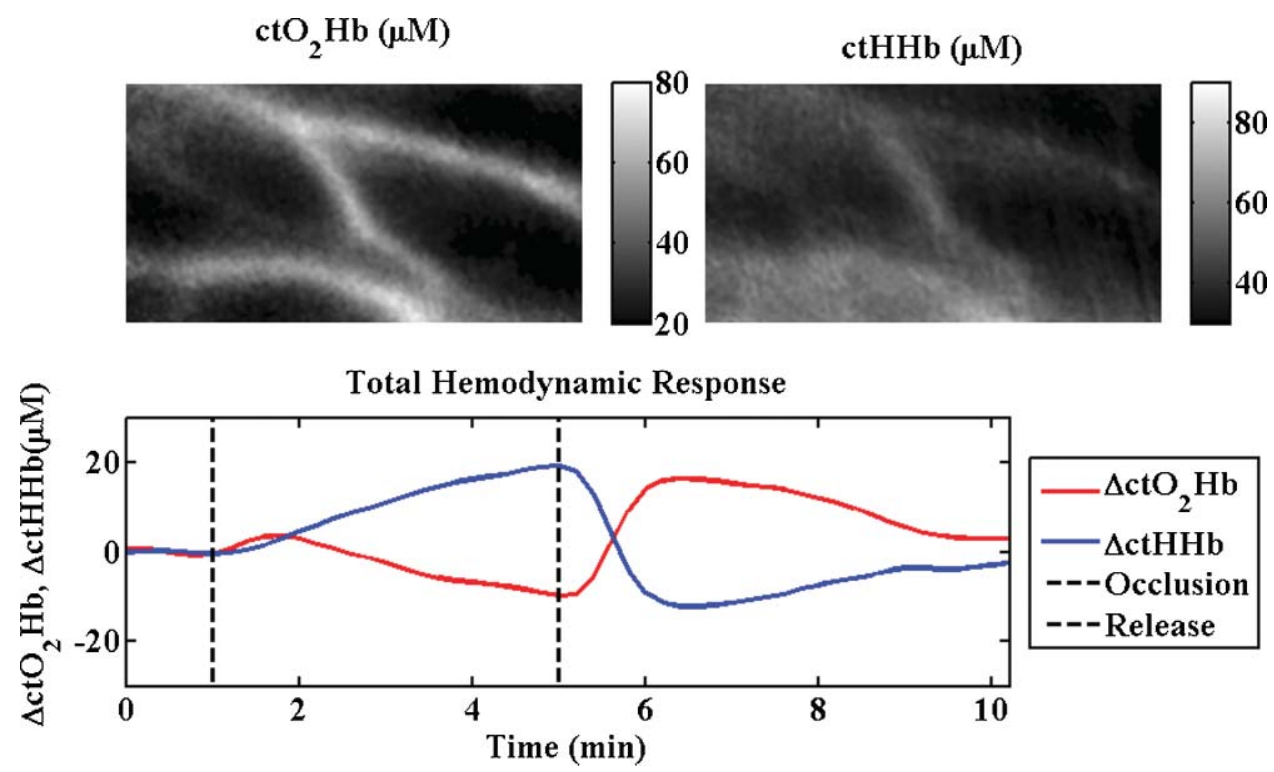

Fig. 7 Arterial occlusion of the wrist. Reduced spectral content improves temporal resolution for hemodynamic imaging. A human wrist is imaged using SFDI with a bicep arterial occlusion at $t=1 \mathrm{~min}$ and release at $t=5 \mathrm{~min}$. SFDI generates maps of hemodynamic changes over time. An $\mathrm{ROI}$ from the image is selected, and the mean changes in $\mathrm{ctO}_{2} \mathrm{Hb}$ and $\mathrm{ctHHb}$ values from baseline are plotted over the course of the occlusion experiment (Video: QuickTime, 416 kB). [URL: http://dx.doi.org/10.1117/1.3523373.1].

were assumed to be $50 \%$ and $2 \%$, respectively. Becuase SFDI is a nonscanning method, the reduced spectral content enables rapid 2-D imaging of the chromophore changes (Fig. 7 and associated video ). An ROI can be selected to analyze spatially variant responses of the tissue (i.e., vein versus microvasculature). In this case, the whole image is analyzed to show the response to the arterial occlusion. After blood flow is occluded at $t=1 \mathrm{~min}$, continued tissue oxygen consumption produces an increase in $\mathrm{ctHHb}$ at the expense of $\mathrm{ctO}_{2} \mathrm{Hb}$. The release of the occlusion results in rapid tissue reperfusion. As new blood rushes into the region to compensate for the cuff-induced hypoxia, $\mathrm{ctO}_{2} \mathrm{Hb}$ and $\mathrm{ctHHb}$ increase and decrease, respectively. The values overshoot initially and then return to baseline after recovery as expected. There are still some residual lines in the reconstructed maps at certain time points due to motion artifacts even despite the rapid acquisition.

\section{Discussion}

These studies were carried out to evaluate spectral design considerations for SFDI systems. We have established that a fast hemodynamic system can be built using two wavelengths and that we can perform fitting for hemoglobin with basic assumptions regarding water and lipid. A $670 \mathrm{~nm} / 850 \mathrm{~nm}$ combination and a $730 \mathrm{~nm} / 850 \mathrm{~nm}$ show similar mean error, but the former has much higher stability. Although, the latter can be used effectively by averaging discrete time points, this approach means sacrificing temporal resolution. Water fractions can change spatially in dynamic measurements due to event onsets such as edema and necrosis, and thus, it is important to account for this while fitting. Thus, it may be an important design consideration to fit for other chomophores if the tissue of interest has high variability in water or lipid content and accuracy is paramount. This can be achieved by measuring data at additional
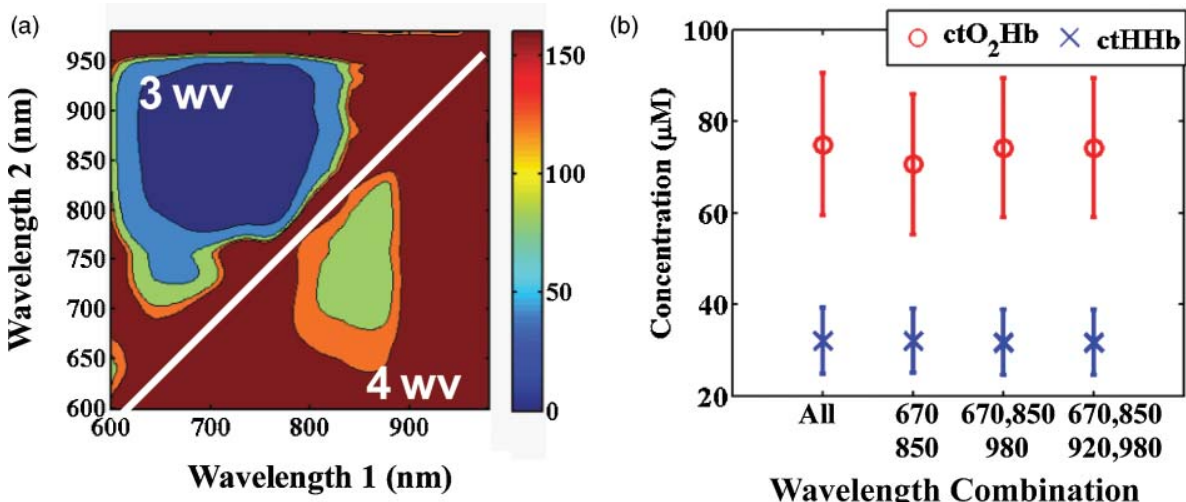

Fig. 8 Multiple wavelength fitting. (a) Condition number plot for wavelength pairs of three wavelengths $(\mathrm{ctO} 2 \mathrm{Hb}, \mathrm{ctHH}$ $\left(\mathrm{ctO}_{2} \mathrm{Hb}, \mathrm{ctHHb}\right.$, water, lipid); $980 \mathrm{~nm}$ is fixed to fit for water in three wavelength fit and $920 \mathrm{~nm}$ is fixed to fit for lipid in four wavelength fit. (b) In vivo data show that optimized three and four wavelength sets can be used to fit for water and lipid and retain accuracy in hemodynamic fits. 

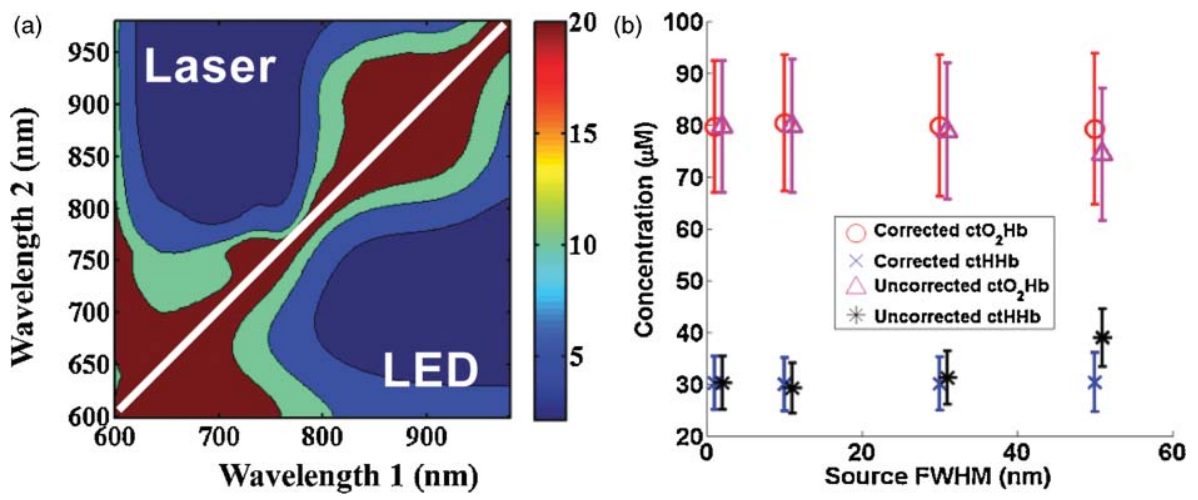

Fig. 9 (a) Condition number $(\kappa)$ for $\mathrm{ctO}_{2} \mathrm{Hb}$ and $\mathrm{ctHHb}$ for laser sources (discrete-wavelength) and LED (FWHM = $30 \mathrm{~nm}$ ). (b) The use of LEDs has minimal affect on reconstructed chromophores but can be corrected for with knowledge of source.

wavelengths above $900 \mathrm{~nm}$, typically, the wavelength region in the NIR most sensitive to lipid and water. A three-wavelength analysis of the condition number for $\mathrm{ctO}_{2} \mathrm{Hb}$, ctHHb, and $\mathrm{ctH}_{2} \mathrm{O}$ shows that the appropriate region for pairwise hemodynamic imaging is much reduced [Fig. 8(a)]. The optimal region includes a wavelength between 650 and $800 \mathrm{~nm}$ and a second wavelength between 800 and $950 \mathrm{~nm}$. The local minima are at 720 and $870 \mathrm{~nm}$ when a single wavelength is fixed at $980 \mathrm{~nm}$ to fit for water. If this concept is extended to include lipid and a fourth wavelength $(920 \mathrm{~nm})$, the optimal region is reduced further [Fig. 8(a)]. The optimal region includes a wavelength between 670 and $800 \mathrm{~nm}$ and second wavelength between 800 and $900 \mathrm{~nm}$. The local minima are at 730 and $850 \mathrm{~nm}$. In the multiple-wavelength cases, the absolute value of the condition number increases dramatically. However, the plot is a metric to show relative fits without having a direct correlation with percent error in fits. The two sets of wavelength combinations $(670 \mathrm{~nm} / 850 \mathrm{~nm} / 980 \mathrm{~nm}$ and $670 \mathrm{~nm} / 850 \mathrm{~nm} / 930 \mathrm{~nm} / 980$ $\mathrm{nm}$ ) were fit for the in vivo case. The quality of the hemodynamic fit matched the full 34-wavelength fit using these reduced spectral parameters [Fig. 8(b)]. The two additional wavelengths at $>900 \mathrm{~nm}$ can help the quality of hemodynamic fitting and eliminate the need for assumptions. Thus, a three- or four-wavelength design is an appropriate solution when the desire is to fit for four chromophores.

Spectral selectivity can also be achieved by using discrete light sources. Thus, one approach to achieve faster hemodynamic imaging could be to use discrete light sources to illuminate the sample. Multiple light sources can also be paired with a multiple-camera detection scheme couple with filters to differentiate light sources. For example, two discrete-wavelength lasers diodes (LD) can be implemented and captured by two cameras, each sensitive to a particular wavelength. Lasers can provide discrete spectral content but are subject to special clinical precautions due to safety reasons. The availability of broader bandwidth and high-power light-emitting diodes (LEDs) has expanded the options for a possible light source. The drawback is that they do not provide the same spectral resolution. The basis spectra used in the analysis thus far for chromophore fits are values at discrete wavelengths. Thus, in order to simulate laser-based measurements, the basis spectra were not altered. However, in order to simulate an LED, the basis spectra for each chromophore were convolved with a 30-nm bandwidth Gaussian filter. The effect on the condition number for hemodynamic fitting was then evaluated for wavelength combinations [Fig. 9(a)]. The minimum condition number is unchanged ( $<5 \%$ variation) based on the choice of a LD or LED [Fig. 9(a)]. The broader spectral bandwidth of a LED smooth out sharp features in the plot, but the lowest condition number is the same in both the laser and LED cases. Simulated data for various spectral bandwidths shows that the light source FWHM can have a small impact on results [Fig. 9(b)]. For example, all sources up to $30 \mathrm{~nm}$ FWHM show a $<5 \%$ error in reconstructed values whether or not the spectra are corrected. The data indicate that correction for spectral characteristics is not necessary until $30 \mathrm{~nm}$ FWHM at the optimized wavelengths. However, it is prudent while data processing to correct for the source spectra in order to ensure an accurate fit. On the basis of this, either light source is appropriate for a system design. LEDs smooth out sharp spectral features and have the potential to be more insensitive to any differences in basis spectra used for data processing. Variability in basis spectra has been shown to cause significant errors in chromophore fits. ${ }^{26,27}$

\section{Conclusion}

This work has shown that the tissue concentration of oxy- and deoxy-hemoglobin can be measured accurately using two wavelengths as long as proper assumptions are made regarding tissue water and lipid. Simulations and in vivo results suggest that condition number analysis is a first-order approximation for chromophore fits in tissue that does not account for confounding chromophores, such as water or lipids. On the basis of these findings, the optimal wavelength pair with the best single measurement stability is 670 and $850 \mathrm{~nm}$, assuming a 50\% water-volume fraction and a $2 \%$ lipid-volume fraction. These assumptions can be varied based on measurement location or tissue type if there is prior knowledge of expected chromophore values. However, completely ignoring these contributions can lead to errors of $>10 \%$ in hemodynamic fits for some wavelength combinations. The reduction in spectral content also significantly enhances SFDI temporal resolution, allowing for spatially resolved measurements of fast hemodynamic events. Additional wavelengths can be added to quantify water and lipid. Condition number analysis is more valid in this case because the contribution of other chromophores in vivo is less significant. Spectral data of in vivo 
measurements confirm that two additional wavelengths can also lead to accurate hemodynamic fits by correcting for water and lipid fractions. It has been shown variability in extinction coefficients can also cause error in chromophore reconstructions. ${ }^{27}$ A broad featured light source, such as an LED, can be used to eliminate sharp spectral features that cause uncertainty in shape of extinction coefficient spectra. In this case, LEDs and LDs can be modeled in such a way that the accuracy of optical property fits is unaffected by the source bandwidth. Other improvements in the accuracy of SFDI calculations include correcting for surface curvature ${ }^{28}$ and applying tomographic algorithms. ${ }^{29}$ We expect that hardware advances can further enhance temporal resolution. Instruments can also be designed using multiple spectral sources and matched with multiple detectors to further improve temporal resolution. Also, high-power sources, such as LEDs, can dramatically increase light throughput and reduce camera exposure times. These advances, in combination with reduced spectral content, are expected to lead to designs for fast and accurate hemodynamic imaging platforms that can be used in various settings on tissues that span a range of sizes and shapes.

\section{Acknowledgments}

This research was made possible by the Laser Microbeam and Medical Program (LAMMP), a NIH Biomedical Technology Resource, Grant No. P41-RR01192; the National Cancer Institute, Grant No. R21-CA129758; the Beckman Foundation; and the Military Photomedicine Program, AFOSR Grant No. FA9550-08-1-0384.

\section{References}

1. B. J. Tromberg, A. Cerussi, N. Shah, M. Compton, A. Durkin, D. Hsiang, J. Butler, and R. Mehta, "Imaging in breast cancer: diffuse optics in breast cancer: detecting tumors in pre-menopausal women and monitoring neoadjuvant chemotherapy," Breast Cancer Res. 7(6), 279-285 (2005).

2. B. J. Tromberg, B. W. Pogue, K. D. Paulsen, A. G. Yodh, D. A. Boas, and A. E. Cerussi, "Assessing the future of diffuse optical imaging technologies for breast cancer management." Med. Phys. 35(6), 24432451 (2008).

3. S. H. Tseng, A. Grant, and A. J. Durkin, "In vivo determination of skin near-infrared optical properties using diffuse optical spectroscopy," $J$. Biomed. Opt. 13(1), 014016 (2008).

4. D. A. Boas, A. M. Dale, and M. A. Franceschini, "Diffuse optical imaging of brain activation: approaches to optimizing image sensitivity, resolution, and accuracy," Neuroimage 23(Suppl 1), S275-288 (2004).

5. G. Yu, T. Durduran, G. Lech, C. Zhou, B. Chance, E. R. Mohler, and A. G. Yodh, "Time-dependent blood flow and oxygenation in human skeletal muscles measured with noninvasive near-infrared diffuse optical spectroscopies," J. Biomed. Opt. 10(2), 024027 (2005).

6. D. T. Delpy, M. Cope, P. ven der Zee, S. Arridge, S. Wray, and J. Wyatt, "Estimation of optical pathlength through tissue from direct time of flight measurement." Phys. Med. Biol. 33(12), 1433-1442 (1988).

7. K. Uludag, M. Kohl, J. Steinbrink, H. Obrig, and A. Villringer, "Cross talk in the Lambert-Beer calculation for near-infrared wavelengths estimated by Monte Carlo simulations," J. Biomed. Opt. 7(1), 51-59 (2002).

8. K. Uludag, J. Steinbrink, A. Villringer, and H. Obrig, "Separability and cross talk: optimizing dual wavelength combinations for near-infrared spectroscopy of the adult head," Neuroimage 22(2), 583-589 (2004).

9. S. Umeyama and T. Yamada, "New cross-talk measure of near-infrared spectroscopy and its application to wavelength combination optimization," J. Biomed. Opt. 14(3), 034017 (2009).
10. H. Sato, M. Kiguchi, F. Kawaguchi, and A. Maki, "Practicality of wavelength selection to improve signal-to-noise ratio in near-infrared spectroscopy," Neuroimage 21(4), 1554-1562 (2004).

11. G. Strangman, M. A. Franceschini, and D. A. Boas, "Factors affecting the accuracy of near-infrared spectroscopy concentration calculations for focal changes in oxygenation parameters," Neuroimage 18(4), 86579 (2003).

12. Y. Yamashita, A. Maki, and H. Koizumi, "Wavelength dependence of the precision of noninvasive optical measurement of oxy-, deoxy-, and total-hemoglobin concentration," Med. Phys. 28(6), 1108-1114 (2001).

13. A. Corlu, R. Choe, T. Durduran, K. Lee, M. Schweiger, S. R. Arridge, E. M. Hillman, and A. G. Yodh, "Diffuse optical tomography with spectral constraints and wavelength optimization," Appl. Opt. 44(11), 2082-2093 (2005).

14. A. Corlu, T. Durduran, R. Choe, M. Schweiger, E. M. Hillman, S. R. Arridge, and A. G. Yodh, "Uniqueness and wavelength optimization in continuous-wave multispectral diffuse optical tomography," Opt. Lett. 28(23), 2339-2341 (2003).

15. M. E. Eames, J. Wang, B. W. Pogue, and H. Dehghani, "Wavelength band optimization in spectral near-infrared optical tomography improves accuracy while reducing data acquisition and computational burden," J. Biomed. Opt. 13(5), 054037 (2008).

16. F. E. Schmidt, J. C. Hebden, E. M. Hillman, M. E. Fry, M. Schweiger, H. Dehghani, D. T. Delpy, and S. R. Arridge, "Multiple-slice imaging of a tissue-equivalent phantom by use of time-resolved optical tomography," Appl. Opt. 39(19), 3380-3387 (2000).

17. H. P. Tuan, C. Olivier, B. F. Joshua, A. Eric, and J. T. Bruce, "Broad bandwidth frequency domain instrument for quantitative tissue optical spectroscopy," Rev. Sci. Instrum. 71(6), 2500-2513 (2000).

18. D. J. Cuccia, F. Bevilacqua, A. J. Durkin, and B. J. Tromberg, "Modulated imaging: quantitative analysis and tomography of turbid media in the spatial-frequency domain," Opt. Lett. 30(11), 1354-1356 (2005).

19. D. J. Cuccia, F. Bevilacqua, A. J. Durkin, and B. J. Tromberg, "Quantitation and mapping of tissue optical properties using modulated imaging," J. Biomed. Opt. 14(2), 024012 (2009).

20. A. Bassi, D. J. Cuccia, A. J. Durkin, and B. J. Tromberg, "Spatial shift of spatially modulated light projected on turbid media," J. Opt. Soc. Am. A. 25(11), 2833-2839 (2008).

21. R. L. van Veen, H. J. Sterenborg, A. Pifferi, A. Torricelli, E. Chikoidze, and R. Cubeddu, "Determination of visible near-IR absorption coefficients of mammalian fat using time- and spatially resolved diffuse reflectance and transmission spectroscopy,"J. Biomed. Opt. 10(5), 054004 (2005).

22. W. G. Zijlstra, A. Buursma, and W. P. Meeuwsen-van der Roest, "Absorption spectra of human fetal and adult oxyhemoglobin, de-oxyhemoglobin, carboxyhemoglobin, and methemoglobin," Clin. Chem. 37(9), 1633-1638 (1991).

23. S. R. Arridge and W. R. Lionheart, "Nonuniqueness in diffusion-based optical tomography," Opt. Lett. 23(11), 882-884 (1998).

24. R. M. Doornbos, R. Lang, M. C. Aalders, F. W. Cross, and H. J. Sterenborg, "The determination of in vivo human tissue optical properties and absolute chromophore concentrations using spatially resolved steadystate diffuse reflectance spectroscopy," Phys. Med. Biol. 44(4), 967-981 (1999).

25. R. F. Reinoso, B. A. Telfer, and M. Rowland, "Tissue water content in rats measured by desiccation," J. Pharmacol. Toxicol. Methods. 38(2), 87-92 (1997).

26. J. G. Kim and H. Liu, "Variation of haemoglobin extinction coefficients can cause errors in the determination of haemoglobin concentration measured by near-infrared spectroscopy," Phys. Med. Biol. 52(20), 6295-6322 (2007).

27. B. Brendel and T. Nielsen, "Selection of optimal wavelengths for spectral reconstruction in diffuse optical tomography," J. Biomed. Opt. 14(3), 034041 (2009).

28. S. Gioux, A. Mazhar, D. J. Cuccia, A. J. Durkin, B. J. Tromberg, and J. V. Frangioni, "Three-dimensional surface profile intensity correction for spatially modulated imaging," J. Biomed. Opt. 14(3), 034045 (2009).

29. S. D. Konecky, A. Mazhar, D. Cuccia, A. J. Durkin, J. C. Schotland, and B. J. Tromberg, "Quantitative optical tomography of sub-surface heterogeneities using spatially modulated structured light," Opt. Express 17(17), 14780-14790 (2009). 